Review article

\title{
fNIRS response during walking - Artefact or cortical activity? A systematic review
}

\author{
R. Vitorio ${ }^{\mathrm{a}, \mathrm{b}}$, S. Stuart ${ }^{\mathrm{a}, \mathrm{c}}$, L. Rochester ${ }^{\mathrm{a}, \mathrm{c}}$, L. Alcock ${ }^{\mathrm{a}}$, A. Pantall ${ }^{\mathrm{a}, *}$

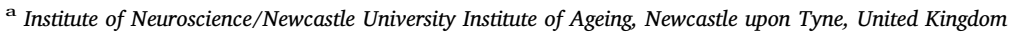 \\ b Universidade Estadual Paulista (UNESP), Instituto de Biociências, Campus Rio Claro, Brazil \\ ${ }^{c}$ The Newcastle upon Tyne NHS Foundation Trust, Newcastle upon Tyne, United Kingdom
}

\section{A R T I C L E I N F O}

\section{Keywords:}

Gait

Complex walking

Dual task

Cortical activation

fNIRS

\begin{abstract}
A B S T R A C T
This systematic review aims to (i) evaluate functional near infrared spectroscopy (fNIRS) walking study design in young adults, older adults and people with Parkinson's disease (PD); (ii) examine signal processing techniques to reduce artefacts and physiological noise in fNIRS data; and (iii) provide evidence-based recommendations for fNIRS walking study design and signal analysis techniques. An electronic search was undertaken. The search request detailed the measurement technique, cohort and walking task. Thirty-one of an initial yield of 73 studies satisfied the criteria. Protocols and methods for removing artefacts and noise varied. Differences in fNIRS signals between studies were found in rest vs. walking, speed of walking, usual vs. complex walking and easy vs. difficult tasks. In conclusion, there are considerable technical and methodological challenges in conducting fNIRS studies during walking which can introduce inconsistencies in study findings. We provide recommendations for the construction of robust methodologies and suggest signal processing techniques implementing a theoretical framework accounting for the physiology of haemodynamic responses.
\end{abstract}

\section{Introduction}

Walking involves dynamic interactions between neuronal structures to co-ordinate the contraction of multiple muscles to successfully navigate complex environments. The ability to cognitively process the surrounding environment and formulate appropriate locomotor plans for navigation can be compromised with ageing and in neurodegenerative disorders such as Parkinson's disease (PD). Studying the changes due to ageing and PD, in which a wide range of progressive walking and cognitive problems are common, may provide insight into the links between cortical activation, cognitive processes and locomotion as well as the potential for the development of new treatments and interventions.

Gait impairment occurs early in PD and evidence suggests that cognitive dysfunction has a prominent role in gait deficits (Galna et al., 2015; Lord et al., 2014). More pronounced walking impairments are evident in both older adults and PD during complex walking (Shumway-Cook and Woollacott, 2007; Vitorio et al., 2014). Gait impairments increase the risk of falls (Lord et al., 2016; Montero-Odasso et al., 2012), with negative consequences to quality of life, independence and health care costs (Stevens et al., 2006). Recording cortical activity during the completion of complex tasks is critical to enhance our understanding of the control of human locomotion and also age- and PD-related changes in mobility.

Functional magnetic resonance imaging (fMRI) studies using gait imagery, have identified cortical areas associated with control of human locomotion. These include the prefrontal cortex, supplementary motor area, premotor cortex, primary motor cortex, primary somatosensory cortex, and sensorimotor cortex (Bakker et al., 2008; Hamacher et al., 2015; la Fougere et al., 2010). While fMRI is considered the gold standard for imaging of the brain in stationary situations, it has limitations for the study of locomotion as participants cannot actually walk in the MRI scanner. Important aspects related to gait control are therefore absent in fMRI studies, such as muscle activity and sensory input from movement (e.g. optic flow and proprioception).

Recent technological advances have enabled monitoring of cortical activity during walking using electroencephalography (EEG), positronemission-tomography (PET) and functional near infrared spectroscopy (fNIRS) devices (Hamacher et al., 2015). fNIRS provides an indirect method for assessing cortical activity through the haemodynamic response of the brain (Maki et al., 1995) and has been validated against fMRI for motor and cognitive tasks (Cui et al., 2011). The first study reporting using fNIRS technology to examine cortical activity during walking was published 16 years ago (Miyai et al., 2001). Following this

\footnotetext{
* Corresponding author at: aInstitute of Neuroscience/Newcastle University Institute of Ageing, Newcastle upon Tyne, United Kingdom.

E-mail address: annette.pantall@newcastle.ac.uk (A. Pantall).
} 
seminal paper, the number of published studies investigating cortical activity with fNIRS has increased exponentially. While fNIRS offers many advantages over other methods including ease of equipment setup and lower costs, the challenge remains of separating the physiological signal representing cortical activity from the noise and artefact components. Systemic physiological changes (heart rate, blood pressure, Mayer waves, respiration, and muscle activity) may be twice as large as those changes arising from the task itself (Boas et al., 2004). Motion and physiological artefacts are particularly problematic during walking and talking activities. Talking results in task related low frequency artefacts with a frequency similar to the haemodynamic response and is more pronounced in anterior channels (prefrontal cortex) (Brigadoi et al., 2014). Currently, there is no standard method for identification and correction of these artefacts in walking studies. Verbal tasks may also result in hypocapnia leading to decreased cerebral blood flow and cerebral oxygenation with a consequential reduction in oxygenated haemoglobin $\left(\mathrm{HbO}_{2}\right)$ (Scholkmann et al., 2013). An additional consideration when processing fNIRS signals is the time-lag of 4-7 s between cortical activity and the haemodynamic response (Cui et al., 2010; Tong and Frederick, 2010). Further, most fNIRS systems only measure attenuation of light rather than absolute changes in haemoglobin, therefore appropriate baseline measures are required (Izzetoglu et al., 2007; Kassab et al., 2015). Another point to consider is that the depth at which $\mathrm{HbO}_{2}$ and deoxygenated haemoglobin (HbR) is assessed is dependent on the distance between optodes (Scholkmann and Wolf, 2012). The method used to analyse the fNIRS signal and specifically the differential path length factor (DPF) selected will affect the estimated changes in $\mathrm{HbO}_{2}$ and $\mathrm{HbR}$ (Scholkmann et al., 2014b). The values of DPF are both age and wavelength dependent and display high intersubject variability (Duncan et al., 1996; Duncan et al., 1995). The mean optical path length also varies spatially and increases with depth of the brain surface (Nakamura et al., 2016). Another factor is what outcome measures are selected for statistical analysis, for example $\mathrm{HbO}_{2}, \mathrm{HbR}$, total haemoglobin or a combination. $\mathrm{HbO}_{2}$ has been reported to be better correlated to the fMRI blood oxygenation level signal and therefore a more accurate indicator of cortical activity (Strangman et al., 2002). A further decision is what measurement value should be used - mean, maximum, minimum, number of peaks or a combination? The mean is considered to be less prone to contamination by artefacts although less sensitive to the physiological signal. However, studies using fNIRS for brain-machine interface have reported inclusion of several measures (mean, maximum, sum of maxima) results in improved identification (Khan and Hong, 2015). Study design, signal processing methods and analysis of fNIRS signals are therefore essential elements in ensuring accurate estimation of the cortical activity from the recorded fNIRS signal.

Previous fNIRS reviews have described the history of fNIRS (Ferrari and Quaresima, 2012), modelling and analysis of fNIRS (Kamran et al., 2016; Orihuela-Espina et al., 2010), comparison of patterns of cortical activity using a variety of imaging techniques in walking studies (Hamacher et al., 2015; Holtzer et al., 2014) and methodological approaches in postural and walking studies (Herold et al., 2017). However, no review has assessed different protocols and signal analysis techniques employed in fNIRS walking studies and how these relate to conflicting findings. The aims of this systematic review are to: (i) evaluate fNIRS walking study design in young adults, older adults and people with $\mathrm{PD}$; (ii) examine signal processing techniques to reduce artefacts and physiological noise in fNIRS data; and (iii) provide evidence-based recommendations for fNIRS walking study design and signal analysis techniques.

\section{Methods}

\subsection{Search strategy}

Two of the authors (SS, LA) created a search strategy to identify all
Table 1

Search terms and synonyms used for each search field.

\begin{tabular}{|c|c|c|c|}
\hline Measurement technique & Population & Gait & Dual task \\
\hline $\begin{array}{l}\text { TITLE-ABS-KEY" } \\
\text { "fNIRS" } \\
\text { "functional near infra*" }\end{array}$ & $\begin{array}{l}\text { TITLE-ABS-KEY' } \\
\text { "young" } \\
\text { "healthy" } \\
\text { "old*" } \\
\text { "elder*" } \\
\text { "neurolog*" } \\
\text { "Parkinson*" }\end{array}$ & $\begin{array}{l}\text { TITLE-ABS-KEY' } \\
\text { "walk*" } \\
\text { "gait" } \\
\text { "locomot"” } \\
\text { "ambul*" } \\
\text { "cue" } \\
\text { "obstac*" }\end{array}$ & $\begin{array}{l}\text { TITLE-ABS-KEY' } \\
\text { "dual*" } \\
\text { "cognit*" } \\
\text { "memory" } \\
\text { "pre-frontal*" } \\
\text { "prefrontal*" }\end{array}$ \\
\hline
\end{tabular}

'*' indicates a wildcard and 'TITLE-ABS-KEY' indicates a title, abstract and keyword search.

potentially relevant studies (Table 1). The search strategy included four fields (connected with "AND") with independent search terms. Those terms in the same search field were linked with the conjunction "OR". The first search field focused on the measurement technique of interest to evaluate cortical activity (i.e. fNIRS). There was no restriction regarding the cortical region being assessed. Given the interest of our research group into ageing and PD we limited our search to three populations. The second search field comprised possible synonyms for populations of interest (i.e. young and older adults and people with $\mathrm{PD})$. The third search field included synonyms for usual and complex walking tasks. The fourth search field comprised synonyms for dual (cognitive and/or motor) tasks and included 'prefrontal' to catch dual task (cognitive studies) assessing the PFC due to known associations with cognitive functions such as working memory and planning (Yuan and Raz, 2014). The search terms were matched and exploded with medical subject headings (MeSH) in the separate databases (Embase, Psych-Info, Pubmed and Scopus). Given that fNIRS technology has only been available since the early 1990's (Ferrari and Quaresima, 2012) the search was limited to articles published post 1989 (until December 2016).

(Insert Table 1 here)

Results were downloaded to a citation manager and duplicates were removed. An initial screen was performed by two reviewers (SS, LA) who reviewed the titles and abstracts. A review of the full text was performed if it was not clear whether the study met the eligibility criteria. Additionally sourced articles were acquired by screening reference lists.

\subsection{Inclusion and exclusion criteria}

Articles were included if they reported the use of fNIRS during an active walking task (e.g. continuous walking, obstacle crossing etc.) with or without performance of a dual task (walking with a secondary concomitant cognitive and/or motor task). Only studies that tested healthy young adults, healthy old adults or people with PD were included (studies involving only obese populations, infants and neurological conditions other than PD were excluded). These groups were included as they are key groups to inform role of cortical control in gait, demonstrating age and disease related compensatory and pathological changes. Articles involving disease-specific groups other than PD were included only when a control group was available which was analysed separately from the clinical group. Only articles written in English were considered for review and any case studies, reviews, book chapters, commentaries, discussion papers, editorials or studies for which the full text was unavailable were excluded.

\subsection{Data extraction}

Data was extracted by the reviewers (SS, LA, RV) and synthesised into table format and data entry confirmed by another reviewer (AP). Data extracted included authors, year of publication, demographic, walking task protocol, dual task protocol, data outcomes, signal 


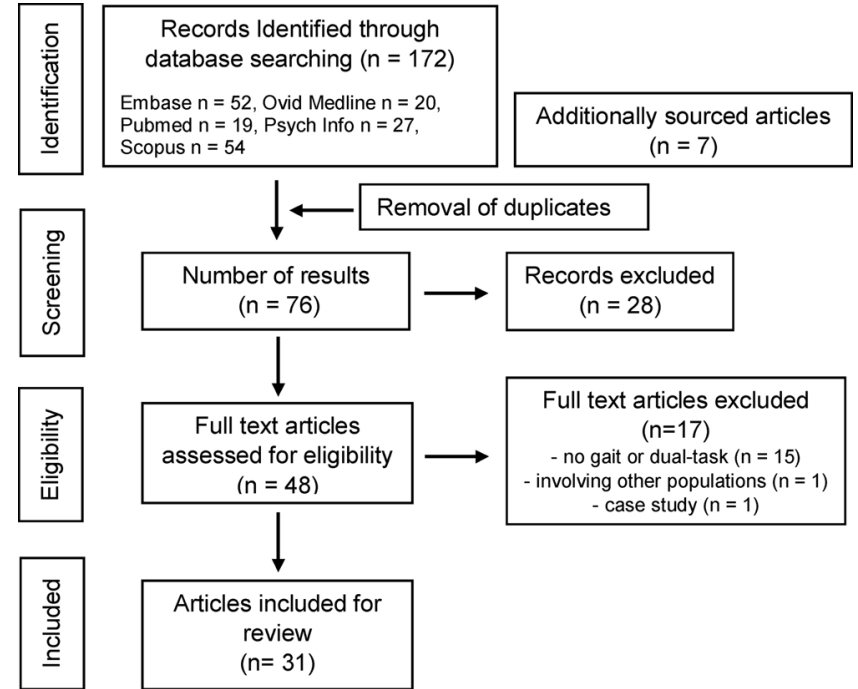

Fig. 1. Flow chart with information through the different phases of the search process.

processing techniques, and pertinent findings.

\section{Results}

\subsection{Study selection}

Fig. 1 provides a flow chart with information regarding the different phases of the search process. The search strategy yielded 172 studies from publication databases and seven more studies were identified by screening of reference lists $(n=179)$. After removal of duplicates $(n=76)$ and further review of the full text, 31 studies were identified for inclusion by consensus of the reviewers. Reasons for exclusion in this phase included: no gait or dual task (Cutini et al., 2011; Deppermann and Vennewald, 2014; Fujimoto et al., 2014; Holper et al., 2012; Huppert et al., 2013; Karim et al., 2012; Kawai et al., 2012; Lareau et al., 2011; Mahoney et al., 2016; Niu et al., 2013; Ono et al., 2014; Piper et al., 2014; Ruocco et al., 2016; Scholkmann et al., 2014a; Wriessnegger et al., 2012), participants other than healthy young adults, healthy older adults or people with PD (Doi et al., 2013), and a case study (Pinti et al., 2015). Fig. 2 demonstrates the cumulative frequency of the reviewed studies which have increased exponentially in the last four years.

\subsection{Study design}

Information relative to participants, tasks, fNIRS devices and cortical areas assessed by all studies included in this systematic review is

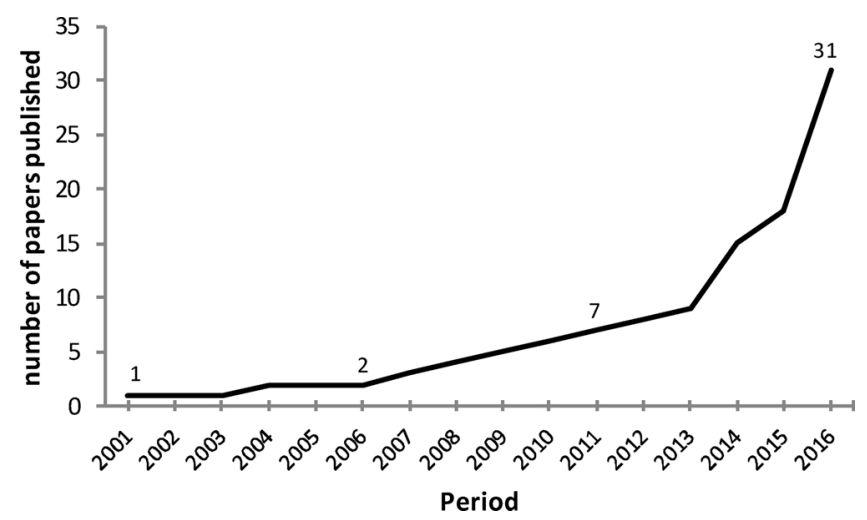

Fig. 2. Cumulative number of research papers published per year using fNIRS to record brain cortical activity during walking in healthy adults and people with PD. presented in Table 2.

\subsubsection{Participants}

Sample size varied between 6 and 348 participants. Distinct groups of healthy young adults were assessed in 16 studies (Atsumori et al., 2010; Beurskens et al., 2014; Fraser et al., 2016; Hill et al., 2013; Holtzer et al., 2011; Kim et al., 2016; Koenraadt et al., 2014; Kurz et al., 2012; Lin and Lin, 2016; Lu et al., 2015; Meester et al., 2014; Metzger et al., 2017; Mirelman et al., 2014; Miyai et al., 2001; Suzuki et al., 2008; Suzuki et al., 2004), healthy older adults in 16 studies (Beurskens et al., 2014; Clark et al., 2014a,b; Eggenberger et al., 2016; Fraser et al., 2016; Harada et al., 2009; Hernandez et al., 2016; Holtzer et al., 2011; Holtzer et al., 2015; Holtzer et al., 2017a; Holtzer et al., 2016; Holtzer et al., 2017b; Maidan et al., 2015; Maidan et al., 2016; Osofundiya et al., 2016; Verghese et al., 2017) and people with PD in three studies (Maidan et al., 2015; Maidan et al., 2016; Nieuwhof et al., 2016). Three studies investigated the effects of ageing (young $v s$. old) on cortical activity during walking (Beurskens et al., 2014; Fraser et al., 2016; Holtzer et al., 2011). Two studies investigated the effects of PD by comparing healthy older adults and people with PD (Maidan et al., 2015; Maidan et al., 2016) (Table 2).

\subsubsection{Walking protocol}

Cortical activity during walking was assessed on a treadmill in 14 studies (Beurskens et al., 2014; Clark et al., 2014a,b; Eggenberger et al., 2016; Fraser et al., 2016; Harada et al., 2009; Kim et al., 2016; Koenraadt et al., 2014; Kurz et al., 2012; Meester et al., 2014; Metzger et al., 2017; Mihara et al., 2007; Miyai et al., 2001; Suzuki et al., 2008; Suzuki et al., 2004) and over-ground in 18 studies (Atsumori et al., 2010; Clark et al., 2014a,b; Hernandez et al., 2016; Hill et al., 2013; Holtzer et al., 2011; Holtzer et al., 2015; Holtzer et al., 2017a; Holtzer et al., 2016; Holtzer et al., 2017b; Lin and Lin, 2016; Lu et al., 2015; Maidan et al., 2015; Maidan et al., 2016; Mirelman et al., 2014; Nieuwhof et al., 2016; Osofundiya et al., 2016; Verghese et al., 2017). Only one study compared cortical activity between treadmill and overground walking (Clark et al., 2014a,b). Four studies investigated the effect of treadmill speed on cortical activity (Harada et al., 2009; Meester et al., 2014; Metzger et al., 2017; Suzuki et al., 2004). Usual walking was assessed in 29 of 31 studies (only Atsumori et al. (2010) and Nieuwhof et al. (2016) compared different dual tasks), motor dual task in four studies (Atsumori et al., 2010; Beurskens et al., 2014; Clark et al., 2014a,b; Lu et al., 2015), cognitive dual task in 20 studies (Beurskens et al., 2014; Clark et al., 2014a,b; Fraser et al., 2016; Hernandez et al., 2016; Hill et al., 2013; Holtzer et al., 2011; Holtzer et al., 2015; Holtzer et al., 2017a; Holtzer et al., 2016; Holtzer et al., 2017b; Lin and Lin, 2016; Lu et al., 2015; Maidan et al., 2016; Meester et al., 2014; Metzger et al., 2017; Mirelman et al., 2014; Nieuwhof et al., 2016; Osofundiya et al., 2016; Verghese et al., 2017), obstacle avoidance in three studies (Clark et al., 2014a,b; Lin and Lin, 2016; Maidan et al., 2016), and precision stepping in two studies (Koenraadt et al., 2014; Osofundiya et al., 2016).All the cognitive dual tasks involved vocalisation, except for Lin et al. (2016) whose dual task involved using a mobile phone and Beurskens et al. (2014) who asked participants to tick boxes on a piece of paper. Twenty-one studies compared cortical activity between usual and dual task walking; the other ten studies reported either usual walking relative to a rest condition or compared different types of dual-task walking. The studies reviewed employed a variety of designs including different trial durations (from 20 to $120 \mathrm{~s}$ ), distance covered during walking trials (from 4.6 to $100 \mathrm{~m}$ ), and number of trial repetitions performed (from two to 15 repetitions) (Table 3). Four studies investigating overground walking assessed straight walks less than $8 \mathrm{~m}$ long (Hill et al., 2013; Holtzer et al., 2011; Holtzer et al., 2015; Holtzer et al., 2017b).

\subsection{3. fNIRS devices and cortical areas assessed}

Fourteen different types of fNIRS devices were used, including two 


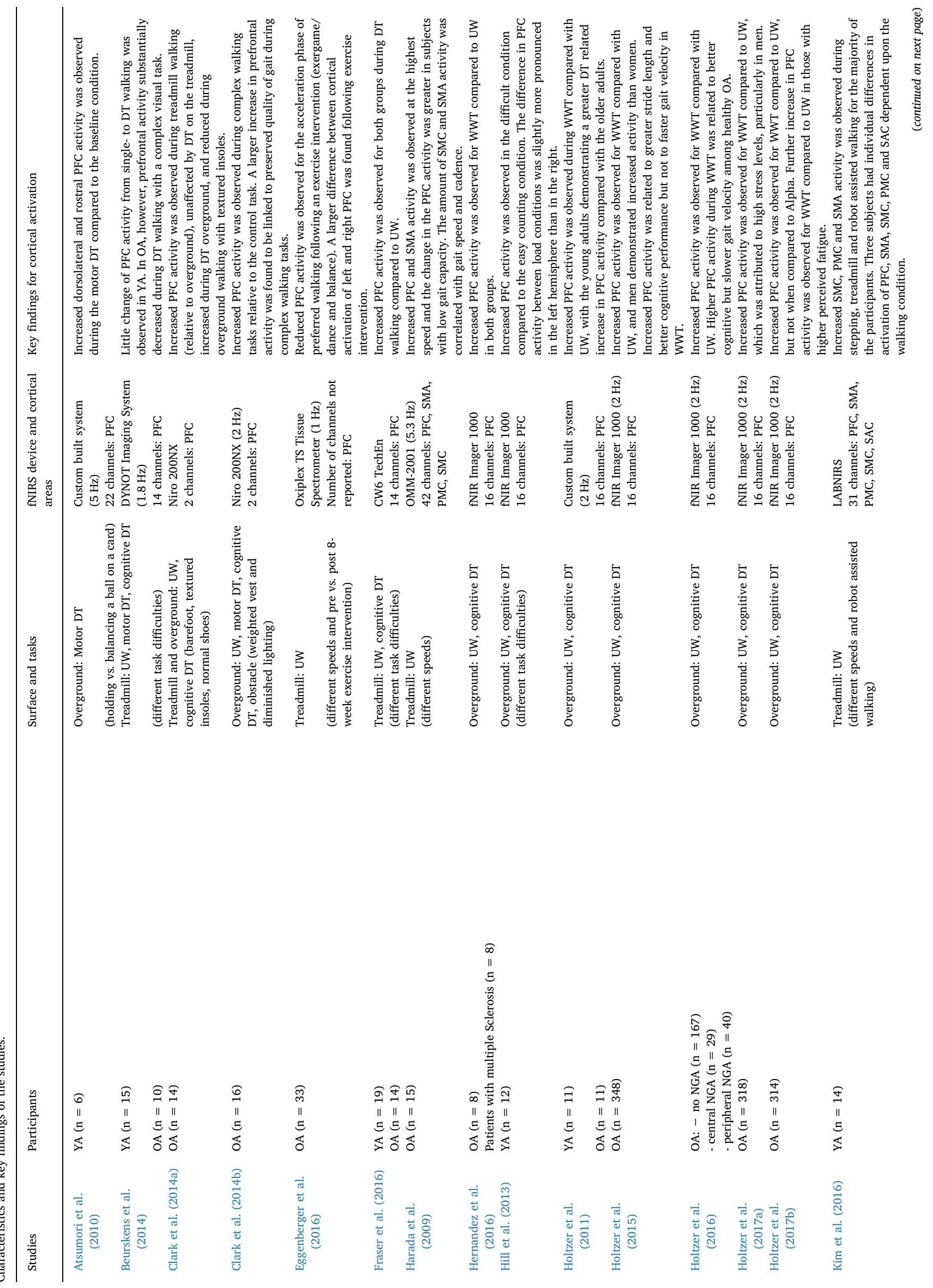




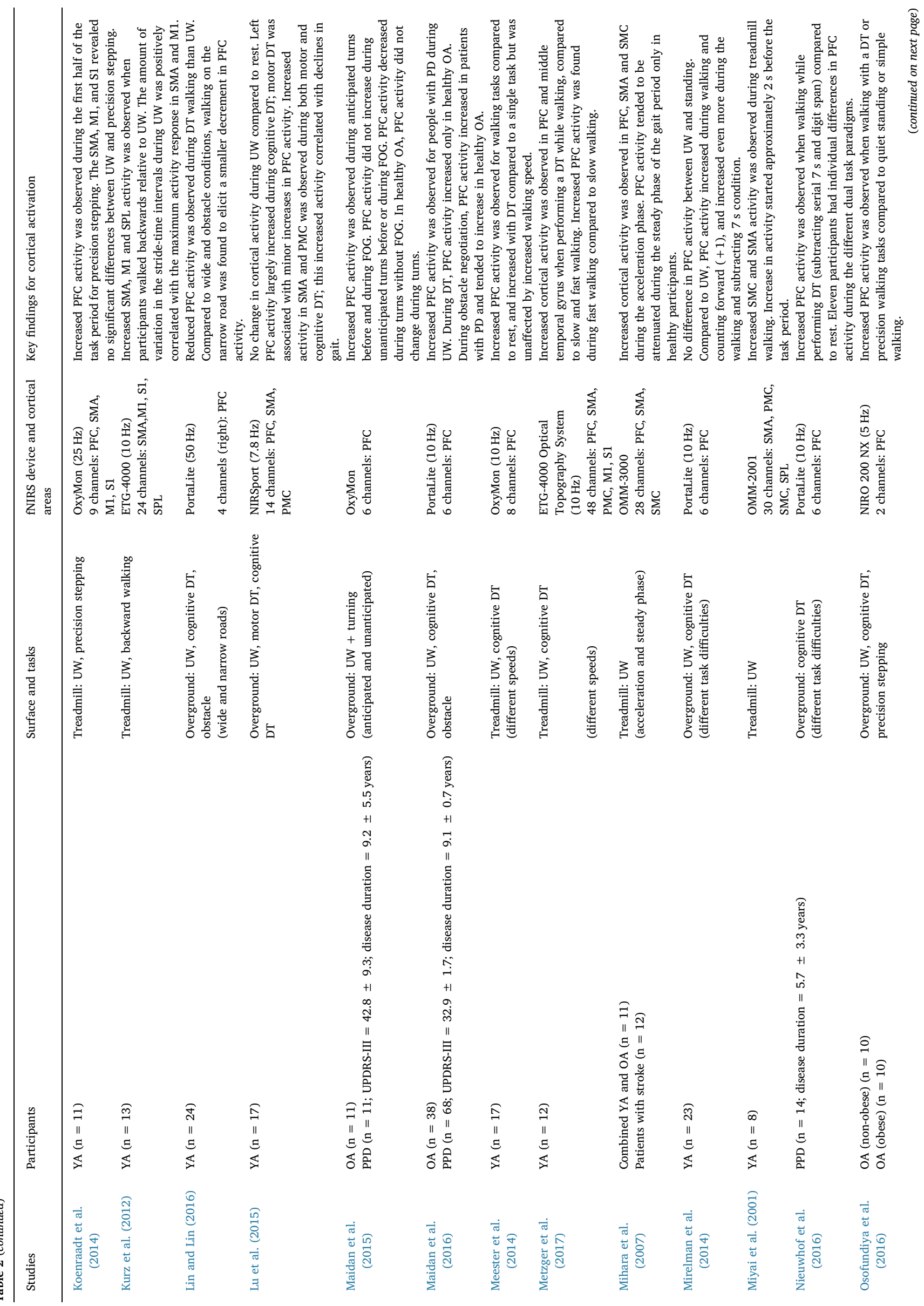


custom built systems (Atsumori et al., 2010; Holtzer et al., 2011) and 12 commercial systems. Twenty-five devices were tethered and six were wireless. The devices were all based on continuous wave technology with both light emitting diodes and laser diodes (von Luhmann, 2014). Sampling frequency varied from 1 to $50 \mathrm{~Hz}$ and number of channels recorded from 2 to 48 (Table 2). The prefrontal cortex was assessed in twenty-nine studies (only Kurz et al. (2012) and Miyai et al. (2001) did not record prefrontal cortex activation), supplementary motor area in nine studies (Harada et al., 2009; Kim et al., 2016; Koenraadt et al., 2014; Kurz et al., 2012; Lu et al., 2015; Metzger et al., 2017; Mihara et al., 2007; Miyai et al., 2001; Suzuki et al., 2008), premotor cortex in seven studies (Harada et al., 2009; Kim et al., 2016; Lu et al., 2015; Metzger et al., 2017; Miyai et al., 2001; Suzuki et al., 2008; Suzuki et al., 2004), sensorimotor cortex in nine studies (Harada et al., 2009; Kim et al., 2016; Koenraadt et al., 2014; Kurz et al., 2012; Metzger et al., 2017; Mihara et al., 2007; Miyai et al., 2001; Suzuki et al., 2008; Suzuki et al., 2004), and superior parietal cortex in two studies (Kurz et al., 2012; Miyai et al., 2001). Overall, ten studies reported the activation of multiple cortical areas and twenty-one studies reported the activation of prefrontal cortex only.

\subsubsection{Signal processing}

A review of fNIRS signal processing techniques is summarised in Table 3. Most studies processed the fNIRS signal over the entire task duration. Only four studies described accounting for the temporal delay of 4-7 s between the task and haemodynamic systemic response by excluding the initial period (Atsumori et al., 2010; Lu et al., 2015; Meester et al., 2014; Mihara et al., 2008).

Sixteen studies applied low pass filters with defined thresholds to remove high frequency components (Hernandez et al., 2016; Holtzer et al., 2011; Holtzer et al., 2015; Holtzer et al., 2017a; Holtzer et al., 2016; Holtzer et al., 2017b; Kim et al., 2016; Koenraadt et al., 2014; Lin and Lin, 2016; Lu et al., 2015; Maidan et al., 2015; Meester et al., 2014; Metzger et al., 2017; Mirelman et al., 2014; Nieuwhof et al., 2016; Verghese et al., 2017). Cut-off frequencies ranged from $0.1 \mathrm{~Hz}$ to $0.2 \mathrm{~Hz}$ with the exception of two studies that selected cut-off frequencies ranging from $0.25-1.25 \mathrm{~Hz}$ (Koenraadt et al., 2014; Meester et al., 2014). Beurskens et al. (2014) applied a pre-colouring filter using the haemodynamic response function, which acts as a low pass filter (Beurskens et al., 2014). Low frequency components were removed with a high pass filter (threshold of $0.01 \mathrm{~Hz}$ ) in four studies (Koenraadt et al., 2014; Kurz et al., 2012; Lu et al., 2015; Maidan et al., 2016). Motion artefacts were generally identified by visual inspection of the signal (Beurskens et al., 2014; Eggenberger et al., 2016; Hernandez et al., 2016; Holtzer et al., 2015; Holtzer et al., 2016; Meester et al., 2014) and/or applying motion artefact rejection algorithms (Beurskens et al., 2014; Hill et al., 2013; Lu et al., 2015; Nieuwhof et al., 2016). Signals containing motion artefacts were either removed or modified. Modification involved applying a spline interpolation algorithm (Beurskens et al., 2014; Nieuwhof et al., 2016) within the movement artefact reduction algorithm (MARA) (Scholkmann et al., 2010) or using wavelet filtering and correlation-based signal improvement (Maidan et al., 2016; Metzger et al., 2017). Methods other than filtering to remove systemic artefacts included wavelet-minimum description length de-trending algorithm (Beurskens et al., 2014; Kim et al., 2016) or using short separation channels as reference channels (Koenraadt et al., 2014). The latter study also recorded blood pressure through a finger cuff to account for systemic oxygenation changes (Koenraadt et al., 2014). Signal drift (along with environmental and equipment noise) was removed through principal component analysis (PCA) or independent component analysis (ICA) in three studies (Holtzer et al., 2011; Kurz et al., 2012; Lu et al., 2015). Atsumori et al. (2010) removed baseline drift through applying a linear trend with regression by least squares to adjust the $\mathrm{HbO}_{2}$ signals. Eggenberger et al. (2016) detrended signals using the $60 \mathrm{~s}$ moving average value. 
Table 3

Signal processing techniques applied by the studies.

\begin{tabular}{|c|c|c|c|}
\hline Studies & Task blocks & General data processing & $\begin{array}{l}\text { Baseline condition, } \\
\text { interoptode distance and } \\
\text { outcomes }\end{array}$ \\
\hline $\begin{array}{l}\text { Atsumori et al. } \\
\qquad(2010)\end{array}$ & $\begin{array}{l}\text { Two sets of alternating task and control walking were } \\
\text { performed (each set included five repetitions of } \\
\text { walking for } 20 \mathrm{~s} \text { for the task and six for the control } \\
\text { condition). }\end{array}$ & $\begin{array}{l}\text { Linear trend of the baseline was removed from task signals. } \\
\text { No filters described. }\end{array}$ & $\begin{array}{l}\text { Baseline: walking while } \\
\text { holding a ball. } \\
\text { Interoptode distance: } 30 \mathrm{~mm} \text {. } \\
\text { fNIRS outcome: } \mathrm{HbO}_{2} \text { and } \mathrm{HbR} \\
\text { (mean value over task period). }\end{array}$ \\
\hline $\begin{array}{l}\text { Beurskens et al. } \\
\text { (2014) }\end{array}$ & $\begin{array}{l}\text { Two repetitions of walking for } 30 \mathrm{~s} \text { were completed for } \\
\text { each condition. }\end{array}$ & $\begin{array}{l}\text { Artefacts were removed using a moving standard deviation and } \\
\text { spline interpolation. Signals were filtered with pre-colouring } \\
\text { method correcting for temporal correlations. Physiological noise } \\
\text { was removed using the wavelet-minimum description length de- } \\
\text { trending algorithm. }\end{array}$ & $\begin{array}{l}\text { Baseline: sitting in a chair. } \\
\text { Interoptode distance: } 22 \mathrm{~mm} \\
\text { and } 25 \mathrm{~mm} \text {. } \\
\text { fNIRS outcome: } \mathrm{HbO}_{2} \text { and } \mathrm{HbR} \\
\text { (mean value over task period). }\end{array}$ \\
\hline Clark et al. (2014a) & $\begin{array}{l}\text { Overground: five consecutive laps around a } 20 \mathrm{~m} \\
\text { circuit; treadmill: } 60-120 \mathrm{~s} \text { of walking. }\end{array}$ & None described. & $\begin{array}{l}\text { Baseline: walking with normal } \\
\text { shoes. } \\
\text { Interoptode distance: } 30 \mathrm{~mm} \text {. } \\
\text { fNIRS outcome: TOI (mean } \\
\text { value over task period). }\end{array}$ \\
\hline Clark et al. (2014b) & Walking five consecutive laps around an $18 \mathrm{~m}$ circuit. & None described. & $\begin{array}{l}\text { Baseline: standing still. } \\
\text { Interoptode distance: } 30 \mathrm{~mm} \text {. } \\
\text { fNIRS outcome: TOI (mean } \\
\text { value over task period). }\end{array}$ \\
\hline $\begin{array}{l}\text { Eggenberger et al. } \\
\text { (2016) }\end{array}$ & $\begin{array}{l}\text { Eight blocks of a } 30 \mathrm{~s} \text { walking period were completed } \\
\text { at preferred and fast pace, with } 30 \mathrm{~s} \text { of rest in between. }\end{array}$ & $\begin{array}{l}\text { Movement artefacts ( }>2.5 \text { and }<2.5 \mu \mathrm{M} \text { HbO2) were removed } \\
\text { using visual inspection and blocks were averaged to minimise } \\
\text { bias from Mayer waves. Signals were detrended and transformed } \\
\text { by subtracting a } 60 \mathrm{~s} \text { moving average. }\end{array}$ & $\begin{array}{l}\text { Baseline: } 1 \mathrm{~min} \text { of very slow } \\
\text { walking }(0.2 \mathrm{~km} / \mathrm{hr} \text { ) } \\
\text { Interoptode distance: four } \\
\text { different distances (not } \\
\text { specified). } \\
\text { fNIRS outcome: } \mathrm{HbO}_{2} \text { (mean } \\
\text { value over task period). }\end{array}$ \\
\hline Fraser et al. (2016) & Six blocks of $120 \mathrm{~s}$ walking for each condition. & None described. & $\begin{array}{l}\text { Baseline: } 5 \text { s standing still. } \\
\text { Interoptode distance: } 28 \mathrm{~mm} \text {. } \\
\text { fNIRS outcome: } \mathrm{HbO}_{2} \text { (mean } \\
\text { value over task period). }\end{array}$ \\
\hline Harada et al. (2009) & $\begin{array}{l}\text { Three repetitions of a } 60 \mathrm{~s} \text { walking period were } \\
\text { completed at different walking speeds. }\end{array}$ & None described. & $\begin{array}{l}\text { Baseline: standing still. } \\
\text { Interoptode distance: } 30 \mathrm{~mm} \text {. } \\
\text { fNIRS outcome: } \mathrm{HbO}_{2} \text { (mean } \\
\text { value over task period). }\end{array}$ \\
\hline $\begin{array}{l}\text { Hernandez et al. } \\
\qquad(2016)\end{array}$ & $\begin{array}{l}\text { Walking for three consecutive loops around a } \\
4 \times 14 \mathrm{ft} \text { walkway, with six straight walks and five } \\
\text { left-sided turns. }\end{array}$ & $\begin{array}{l}\text { Data visually inspected and removed if saturation or dark } \\
\text { current conditions were identified. Signals were low-pass } \\
\text { filtered with a cut-off frequency at } 0.14 \mathrm{~Hz} \text {. }\end{array}$ & $\begin{array}{l}\text { Baseline: standing still. } \\
\text { Interoptode distance: } 25 \mathrm{~mm} \text {. } \\
\text { fNIRS outcome: } \mathrm{HbO}_{2} \text { (mean } \\
\text { value over task period). }\end{array}$ \\
\hline Hill et al. (2013) & $\begin{array}{l}\text { Fifteen repetitions of walking along a } 25 \mathrm{ft} \text { walkway } \\
\text { were performed for each condition. }\end{array}$ & $\begin{array}{l}\text { Physiological noise was removed with a low-pass filter } \\
\text { (threshold not stated). A sliding window motion artefact } \\
\text { rejection routine was also applied. }\end{array}$ & $\begin{array}{l}\text { Baseline: standing still. } \\
\text { Interoptode distance: none } \\
\text { described. } \\
\text { fNIRS outcome: } \mathrm{HbO}_{2} \text { (mean } \\
\text { value over task period). }\end{array}$ \\
\hline Holtzer et al. (2011) & $\begin{array}{l}\text { Six repetitions of walking along a } 15 \mathrm{ft} \text { walkway were } \\
\text { performed for each condition. }\end{array}$ & $\begin{array}{l}\text { Signals were low-pass filtered with a cut-off frequency at } \\
0.14 \mathrm{~Hz} \text {. A combined independent component analysis/principal } \\
\text { component analysis was used to remove noise and signal drifts. }\end{array}$ & $\begin{array}{l}\text { Baseline: standing still. } \\
\text { Interoptode distance: } 25 \mathrm{~mm} \text {. } \\
\text { fNIRS outcome: } \mathrm{HbO}_{2} \\
\text { (maximal value). }\end{array}$ \\
\hline Holtzer et al. (2015) & $\begin{array}{l}\text { Walking for three consecutive loops around a } \\
4 \times 14 \mathrm{ft} \text { walkway, with six straight walks and five } \\
\text { left-sided turns. }\end{array}$ & $\begin{array}{l}\text { Signals were low-pass filtered with a cut-off frequency at } \\
0.14 \mathrm{~Hz} \text {. }\end{array}$ & $\begin{array}{l}\text { Baseline: standing still. } \\
\text { Interoptode distance: } 25 \mathrm{~mm} \text {. } \\
\text { fNIRS outcome: } \mathrm{HbO}_{2} \text { (mean } \\
\text { value over task period). }\end{array}$ \\
\hline Holtzer et al. (2016) & $\begin{array}{l}\text { Walking for three consecutive loops around a } \\
4 \times 14 \mathrm{ft} \text { walkway, with six straight walks and five } \\
\text { left-sided turns. }\end{array}$ & $\begin{array}{l}\text { Signals were low-pass filtered with a cut-off frequency at } \\
0.14 \mathrm{~Hz} \text {. }\end{array}$ & $\begin{array}{l}\text { Baseline: standing still. } \\
\text { Interoptode distance: } 25 \mathrm{~mm} \text {. } \\
\text { fNIRS outcome: } \mathrm{HbO}_{2} \text { (mean } \\
\text { value over task period). }\end{array}$ \\
\hline $\begin{array}{l}\text { Holtzer et al. } \\
\qquad(2017 a)\end{array}$ & $\begin{array}{l}\text { Walking for three consecutive loops around a } \\
4 \times 14 \mathrm{ft} \text { walkway, with six straight walks and five } \\
\text { left-sided turns. }\end{array}$ & $\begin{array}{l}\text { Signals were low-pass filtered with a cut-off frequency at } \\
0.14 \mathrm{~Hz} \text {. }\end{array}$ & $\begin{array}{l}\text { Baseline: standing still. } \\
\text { Interoptode distance: } 25 \mathrm{~mm} \text {. } \\
\text { fNIRS outcome: } \mathrm{HbO}_{2} \text { (mean } \\
\text { value over task period). }\end{array}$ \\
\hline $\begin{array}{l}\text { Holtzer et al. } \\
\qquad(2017 b)\end{array}$ & $\begin{array}{l}\text { Walking for three consecutive loops around a } \\
4 \times 14 \mathrm{ft} \text { walkway, with six straight walks and five } \\
\text { left-sided turns. }\end{array}$ & $\begin{array}{l}\text { Signals were low-pass filtered with a cut-off frequency at } \\
0.14 \mathrm{~Hz} \text {. }\end{array}$ & $\begin{array}{l}\text { Baseline: standing still. } \\
\text { Interoptode distance: } 25 \mathrm{~mm} \text {. } \\
\text { fNIRS outcome: } \mathrm{HbO}_{2} \text { (mean } \\
\text { value over task period). }\end{array}$ \\
\hline Kim et al. (2016) & $\begin{array}{l}\text { Five randomised blocks of } 30 \mathrm{~s} \text { walking followed by } \\
30 \mathrm{~s} \text { rest for stepping and walking. Three blocks of } 60 \mathrm{~s} \\
\text { robot walking followed by } 60 \mathrm{~s} \text { rest. }\end{array}$ & $\begin{array}{l}\text { Gaussian smoothing with a full width at half max of } 2 \mathrm{~s} \text {. } \\
\text { Movement artefacts were removed using the wavelet-minimum } \\
\text { description length de-trending algorithm. }\end{array}$ & $\begin{array}{l}\text { Baseline: resting without } \\
\text { moving. } \\
\text { Interoptode distance: } 30 \mathrm{~mm} \text {. } \\
\text { fNIRS outcome: } \mathrm{HbO}_{2} \text { (mean } \\
\text { value over task period). } \\
\quad \text { (continued on next page) }\end{array}$ \\
\hline
\end{tabular}


Table 3 (continued)

\begin{tabular}{lll}
\hline Studies & Task blocks & General data processing \\
& & $\begin{array}{l}\text { Baseline condition, } \\
\text { interoptode distance and } \\
\text { outcomes }\end{array}$
\end{tabular}

Koenraadt et al. Ten repetitions of $35 \mathrm{~s}$ were performed for each (2014) condition.

Kurz et al. (2012) Two sessions with five repetitions of $30 \mathrm{~s}$ of walking were performed for each condition.

Lin and Lin (2016) For each condition, participants walked along a $20 \mathrm{~m}$ walkway for 60 s.

$\begin{array}{ll}\text { Lu et al. (2015) } & \begin{array}{l}\text { Three repetitions of a } 60 \mathrm{~s} \text { walking periods along a } \\ 5.5 \mathrm{~m} \text { walkway were completed. }\end{array} \\ \begin{array}{c}\text { Maidan et al. } \\ \text { (2015) }\end{array} & \begin{array}{l}\text { Participants walked } 20 \mathrm{~m} \text {, turned } 180^{\circ} \text { and walked in } \\ \text { the opposite direction. Only intervals of } 6 \mathrm{~s} \text { of } \\ \text { consecutive walking and } 180^{\circ} \text { turns were included for } \\ \text { analyses. }\end{array} \\ \begin{array}{c}\text { Maidan et al. } \\ \text { (2016) }\end{array} & \begin{array}{l}\text { Five repetitions of walking along a } 30 \mathrm{~m} \text { walkway for } \\ 30 \mathrm{~s} \text { were performed for each condition. }\end{array}\end{array}$

Four randomised blocks of $10 \mathrm{~s}$ rest, $45 \mathrm{~s}$ walking $(3 \mathrm{~km} / \mathrm{hr}), 15 \mathrm{~s}$ rest, $45 \mathrm{~s}$ walking $(5 \mathrm{~km} / \mathrm{hr}), 15 \mathrm{~s}$ rest, $45 \mathrm{~s}$ DT walking, $15 \mathrm{~s}$ rest. for each condition. performed for each condition.
A bandpass filter $(0.01 \mathrm{~Hz}-1.25 \mathrm{~Hz})$ was applied to the signals. Short separation channels were used to remove haemodynamic changes in superficial tissue layers. An additional low-pass filter with a cut-off frequency of $1 \mathrm{~Hz}$ was applied. The changes were normalised by dividing the change for each individual by the maximum change for that individual.

Data were filtered with a $0.01 \mathrm{~Hz}$ high-pass filter and a $5.0 \mathrm{~s}$ moving average filter. Principal component analyses were applied to reduce physiological noise.

Data were filtered with a low-pass filter with a cut-off frequency of $0.2 \mathrm{~Hz}$.

Signals were bandpass-filtered $(0.01 \mathrm{~Hz}-0.2 \mathrm{~Hz})$. Motion artefacts were removed through principal component analysis and spike rejection.

Signals were low pass filtered with a cut-off frequency at $0.14 \mathrm{~Hz}$.

Data were bandpass filtered $(0.01 \mathrm{~Hz}$ to $0.14 \mathrm{~Hz})$. A wavelet filter and correlation based signal improvement were applied to remove motion artefact.

Signals were filtered with a low pass filter set at $0.67 \mathrm{~Hz}$. A moving average filter with a width of $4 \mathrm{~s}$ was used to smooth the signal.

Channels with large movement or technical artefacts removed. Smaller artefacts corrected with Correlation Based Signal Improvement (CBSI) method. Signals were low-pass filtered with a $5 \mathrm{~s}$ moving average filter.

None described.

Signals were low-pass filtered with a cut-off frequency at $0.14 \mathrm{~Hz}$.

Signals were analysed with SPM99 (Statistical Parametric Mapping software; Wellcome Department of Cognitive Neurology, London, UK).

Moving standard deviation based artefact removal (moving artefact reduction algorithm: MARA) with threshold of 0.45 for $\mathrm{HbO}_{2}$ and 0.18 for HbR. Signals were linearly de-trended and low-pass filtered at $0.1 \mathrm{~Hz}$.

None described.

Signals were analysed with SPM99 (Statistical Parametric Mapping software; Wellcome Department of Cognitive Neurology, London, UK).

Signals were analysed with SPM99 (Statistical Parametric Mapping software; Wellcome Department of Cognitive Neurology, London, UK).
Baseline: standing still. Interoptode distance: $10 \mathrm{~mm}$ and $30 \mathrm{~mm}$

fNIRS outcome: $\mathrm{HbO}_{2}$ and $\mathrm{HbR}$ (mean value over task period).

Baseline: standing still Interoptode distance: $30 \mathrm{~mm}$. fNIRS outcome: $\mathrm{HbO}_{2}$ (maximal value) and $\mathrm{HbR}$ (minimum value).

Baseline: standing still. Interoptode distance: $40 \mathrm{~mm}$. fNIRS outcome: $\mathrm{HbO}_{2}$ (mean, 10th and 90th percentiles and range)

Baseline: standing still.

Interoptode distance: $30 \mathrm{~mm}$. fNIRS outcome: haemoglobin differential $\left(=\mathrm{HbO}_{2}-\mathrm{HbR}\right)$ Baseline: walking $6 \mathrm{~s}$ before a freezing of gait event. Interoptode distance: $35 \mathrm{~mm}$. fNIRS outcome: $\mathrm{HbO}_{2}$ (mean value over task period). Baseline: standing still. Interoptode distance: 30, 35, and $40 \mathrm{~mm}$.

fNIRS outcome: $\mathrm{HbO}_{2}$ (mean value over task period). Baseline: standing still. Interoptode distance: $30 \mathrm{~mm}$. fNIRS outcome: $\mathrm{HbO}_{2}$ and $\mathrm{HbR}$ (mean value over task period) Baseline: standing still; $10 \mathrm{~s}$ at beginning of each block. Interoptode distance: $30 \mathrm{~mm}$. fNIRS outcome: $\mathrm{HbO}_{2}$ (maximal value) and $\mathrm{HbR}$ (minimum value).

Baseline: standing still. Interoptode distance: $30 \mathrm{~mm}$. fNIRS outcome: $\mathrm{HbO}_{2}$ (mean value over task period). Baseline: standing still. Interoptode distance: none described.

fNIRS outcome: $\mathrm{HbO}_{2}$ (mean value over task period). Baseline: standing or sitting still.

Interoptode distance: $30 \mathrm{~mm}$. fNIRS outcome: $\mathrm{HbO}_{2}$ and $\mathrm{HbR}$ (mean value over task period). Baseline: standing still. Interoptode distance: 30, 35, and $40 \mathrm{~mm}$. fNIRS outcome: $\mathrm{HbO}_{2}$ and $\mathrm{HbR}$ (mean value over task period). Baseline: Relative to zero. Interoptode distance: Fpz to Fp3/Fp4.

fNIRS outcome: $\mathrm{HbO}_{2}$ (maximal value).

Baseline: standing still. Interoptode distance: $30 \mathrm{~mm}$. fNIRS outcome: $\mathrm{HbO}_{2}$ (mean value over task period). Baseline: standing still. Interoptode distance: $30 \mathrm{~mm}$. fNIRS outcome: $\mathrm{HbO}_{2}$ and $\mathrm{HbR}$ (mean value over task period). (continued on next page) 
Table 3 (continued)

\begin{tabular}{|c|c|c|c|}
\hline Studies & Task blocks & General data processing & $\begin{array}{l}\text { Baseline condition, } \\
\text { interoptode distance and } \\
\text { outcomes }\end{array}$ \\
\hline $\begin{array}{l}\text { Verghese et al. } \\
\qquad(2017)\end{array}$ & $\begin{array}{l}\text { Walking for three consecutive loops around a } \\
4 \times 14 \mathrm{ft} \text { walkway, with six straight walks and five } \\
\text { left-sided turns. }\end{array}$ & $\begin{array}{l}\text { Signals were low pass filtered with a cut-off frequency at } \\
0.14 \mathrm{~Hz} \text {. }\end{array}$ & $\begin{array}{l}\text { Baseline: standing still. } \\
\text { Interoptode distance: none } \\
\text { described. } \\
\text { fNIRS outcome: } \mathrm{HbO}_{2} \text { (mean } \\
\text { value over task period). }\end{array}$ \\
\hline
\end{tabular}

Acronyms: $\mathrm{HbO}_{2}$, oxygenated haemoglobin; $\mathrm{HbR}$, de-oxygenated haemoglobin; TOI, tissue oxygenation index.

\subsubsection{Interoptode distance}

The interoptode distances selected to measure haemoglobin ranged from a minimum $22 \mathrm{~mm}$ (Beurskens et al., 2014) to a maximum $40 \mathrm{~mm}$ (Lin and Lin, 2016; Maidan et al., 2016; Nieuwhof et al., 2016). The mode was $30 \mathrm{~mm}$, applied by sixteen of the twenty six reviewed studies which reported interoptode distances. Two studies used the Artinis Portalite system which has three different interoptode distances of $30 \mathrm{~mm}, 35 \mathrm{~mm}$ and $40 \mathrm{~mm}$ (Maidan et al., 2016; Nieuwhof et al., 2016).

\subsubsection{Determination of $\mathrm{HbO}_{2}$ and $\mathrm{HbR}$}

All the fifteen studies that described the algorithm to determine $\mathrm{HbO}_{2}$ and $\mathrm{HbR}$ applied the Beer-Lambert Law. However, only two studies specified the DPF used in the algorithm (Koenraadt et al., 2014; Nieuwhof et al., 2016). Koenraadt et al. (2014) selected the values experimentally determined by Duncan et al. (1996) whereas Nieuwhof et al. (2016) applied a DPF of 6 to all participants and regions. A third study reported that they assumed the optical path length was equal for all wavelengths and constants but did not state what values they applied (Atsumori et al., 2010).

\subsubsection{Outcome measures}

Twenty of the reviewed studies analysed only $\mathrm{HbO}_{2}$, eight analysed both $\mathrm{HbO}_{2}$ and HbR haemoglobin (Atsumori et al., 2010; Beurskens et al., 2014; Koenraadt et al., 2014; Meester et al., 2014; Metzger et al., 2017; Miyai et al., 2001; Nieuwhof et al., 2016; Suzuki et al., 2008), two analysed total haemoglobin (Clark et al., 2014a,b) and one study assessed the difference between $\mathrm{HbO}_{2}$ and $\mathrm{HbR}$ (Lu et al., 2015). Twenty six of the reviewed studies analysed the mean of their selected parameters, two studies selected the maximum and minimum (Kurz et al., 2012; Metzger et al., 2017), two studies only the maximum (Holtzer et al., 2011; Osofundiya et al., 2016) and one study analysed the mean, 10th and 90th percentile and the range (Lin and Lin, 2016). Nine studies averaged channels or regions of interest across hemispheres (Clark et al., 2014a,b; Holtzer et al., 2017a; Holtzer et al., 2016; Holtzer et al., 2017b; Koenraadt et al., 2014; Kurz et al., 2012; Maidan et al., 2015; Mirelman et al., 2014; Verghese et al., 2017).

\section{3. fNIRS signal during walking}

This review is focused on the methods (protocol and signal processing) and aims to identify if differences in study findings can be attributed to methodological variations. The key findings are reported in Table 2. As the majority of reviewed papers analysed $\mathrm{HbO}_{2}$ as the outcome measure, further reference to the fNIRS signal relates to $\mathrm{HbO}_{2}$ unless stated otherwise. Most studies reported an increase in fNIRS activity during usual walking compared to a resting condition in the prefrontal cortex, premotor cortex, supplementary motor area, primary motor cortex and primary somatosensory cortex (Harada et al., 2009; Holtzer et al., 2011; Holtzer et al., 2015; Meester et al., 2014; Mihara et al., 2007; Suzuki et al., 2008; Suzuki et al., 2004). However, Lu et al. (2015) who analysed the difference between $\mathrm{HbO}_{2}$ and $\mathrm{HbR}$ and Mirelman et al. (2014) found no change in the prefrontal fNIRS signal; Koenraadt et al. (2014) found decreased fNIRS activity in the supplementary motor area and no change in the prefrontal, primary motor and primary somatosensory cortices. Clark et al. (2014a,b), analysing total haemoglobin, reported greater prefrontal fNIRS signals during treadmill walking compared to overground walking in 14 older adults with mobility deficits. Three of the four studies observed an increase in the fNIRS signal as walking speed increased (Harada et al., 2009; Metzger et al., 2017; Suzuki et al., 2004) whereas Meester et al. (2014) reported no change. Walking modality (treadmill vs. overground) influenced prefrontal activity as assessed by total haemoglobin levels, such that with a dual task no change was observed when walking on a treadmill compared to an increase during overground walking (Clark et al., 2014a,b).

Compared to usual walking, dual task walking was mainly associated with an increased prefrontal fNIRS signal in both young and old (Clark et al., 2014a,b; Hill et al., 2013; Holtzer et al., 2011; Holtzer et al., 2015; Holtzer et al., 2016; Koenraadt et al., 2014; Lu et al., 2015; Maidan et al., 2016; Meester et al., 2014; Mirelman et al., 2014). However, Lin and Lin (2016) observed decreased activity in the prefrontal signal during dual task walking in young adults. The two studies that evaluated cortical regions other than the prefrontal cortex during dual tasking walking reported conflicting results in young adults. While Lu et al. (2015) observed increased fNIRS activity (haemoglobin difference) in the premotor cortex and supplementary motor area, Metzger et al. (2017) observed no change in these regions and the primary motor and somatosensory cortices. The three studies that evaluated the effect of age reported contradictory results. Beurskens et al. (2014) found no change in prefrontal fNIRS signal during dual task walking in young adults and a decrease in older adults whereas Holtzer et al. (2011) observed greater activity for both groups during dual task walking with young adults displaying a larger dual task related increase. Fraser et al. (2016), by contrast, reported more activity for both young and older adults for dual task walking, with no difference between groups. The two reviewed studies from the same research group, that investigated the effects of PD on cortical activation during dual task walking observed altered prefrontal fNIRS signals in people with PD compared to older adults (Maidan et al., 2015; Maidan et al., 2016).

\section{Discussion}

This review examined 31 studies that assessed cortical activity using fNIRS during walking in healthy young adults, healthy older adults and people with PD. This review explicitly targeted: (i) the study design, (ii) signal processing techniques and analysis and; (iii) differences between studies reported in fNIRS signals activity during walking. In summary, most studies involved small sample sizes (15 studies with $\mathrm{n}<15$ ) which makes the results difficult to generalise. Studies used a wide range of fNIRS systems, both tethered and wireless, and various protocol designs making comparisons challenging. Several studies analysed walking over short durations or distances $(<6 \mathrm{~s}$ or $8-10 \mathrm{~m})$ which may influence accuracy when considering haemodynamic response time. Signal processing methods for removing noise and artefacts were varied, often inadequately described and not related to specific tasks. Only two studies described the DPF used for determining haemoglobin levels. A recent review has also reported a lack of specific DPF values 
reported for walking studies (Herold et al., 2017). The majority of studies analysed the change in mean $\mathrm{HbO}_{2}$. Discrepancies between study findings may arise from differing study designs or processing techniques. We have structured our discussion accordingly to integrate technical and methodological challenges with primary research questions.

\subsection{Cortical activity}

\subsubsection{Usual walking}

The majority of studies reported an increase in the fNIRS signal during walking compared to a resting condition (Section 3.3). However, the action of walking per se will increase motion artefact as a result of the head movements present in walking causing decoupling between the optodes and scalp (Brigadoi et al., 2014; Chiarelli et al., 2015; Yamada et al., 2015). A recent EEG study demonstrated that motion artefact increases with walking speed and channels are differentially affected (Kline et al., 2015). Additionally, different inertial properties of fNIRS systems may further modify the motion artefact. Two of the studies that reported no change in the fNIRS signal used wireless systems (Lu et al., 2015; Mirelman et al., 2014), which generate a smaller artefact due to the lower weight of cables pulling on the cap or headband. Koenraadt et al. (2014), using a tethered system, also reported differing results to the majority of the reviewed studies. A possible explanation for this specific case is that Koenraadt's study was the only one that used short separation channels to correct the fNIRS signal for haemodynamic changes in superficial tissue layers. Three of the four studies that assessed the effect of walking speed reported increased fNIRS activity (Harada et al., 2009; Metzger et al., 2017; Suzuki et al., 2004). Meester et al. (2014), who observed no change in fNIRS signal with speed, used an 8 channel system which will generate a smaller motion artefact due to its lower inertia in contrast to the 42-48 channel systems used in the three other studies.

Larger fNIRS signals have been recorded from the prefrontal cortex during treadmill walking compared to over-ground walking (Clark et al., 2014a,b). One explanation is the greater metabolic cost incurred (Parvataneni et al., 2009; Riley et al., 2007). Two overground studies (Holtzer et al., 2011; Holtzer et al., 2015) that reported increased prefrontal activity during walking relative to rest analysed short segments of data, approximately $4-5 \mathrm{~s}$ long. Under these conditions the fNIRS signal could also reflect the planning required for (e.g. upcoming turning or stop) as well as straight walking, accounting in part for the greater activity observed.

Methods to remove motion artefact and systemic noise were varied which will modify the processed fNIRS signal's characteristics. Although Holtzer et al. (2011) and Lu et al. (2015) used ICA/PCA, the latter additionally removed channels with a coefficient of variation greater than $15 \%$ and applied spike rejection to the remaining channels. This could further explain why Holtzer et al. (2011) reported greater fNIRS signals during usual walking compared with resting whereas Lu et al. (2015) observed no change across the two conditions. Another explanatory reason could be that Holtzer et al. (2011) used the maximum change in $\mathrm{HbO}_{2}$ which might include more artefacts compared to the mean value due to the larger amount of noise present in the peaks. $\mathrm{Lu}$ et al. (2015) by contrast analysed the mean difference between $\mathrm{HbO}_{2}$ and HbR.

\subsubsection{Complex walking}

Complex walking, including obstacle avoidance and precision stepping, and dual task walking were mainly associated with increased fNIRS activity in the prefrontal cortex relative to usual walking with two exceptions (Beurskens et al., 2014; Lin and Lin, 2016) (Table 2). Many of the cognitive tasks required the participant to respond verbally, which creates motion artefact. In contrast, Beurskens et al. (2014) and Lin and Lin (2016) used nonverbal tasks which may account for their observation of no signal change. Motion artefacts arising from cognitive linguistic tasks are challenging as they have similar frequencies and amplitudes to the haemodynamic responses (Brigadoi et al., 2014). Wavelet filtering is optimal for removing motion artefacts arising from linguistic tasks (Brigadoi et al., 2014). However, of the 12 studies that used a linguistic task, only one applied wavelet filters to remove artefacts (Maidan et al., 2016). A further consideration during dual task walking is the stress evoked by the walking task which may increase superficial blood flow, via increased autonomic nervous system activity, thereby exaggerating the task effect (Kirilina et al., 2012). This is a particular issue when recording fNIRS from the prefrontal cortex due to greater changes in blood flow to the forehead compared with other cranial regions (Kirilina et al., 2012; Takahashi et al., 2011). Only one study accounted for changes in superficial scalp blood flow externally by using short separation channels in addition to a finger cuff to measure heart rate and systemic blood pressure changes (Koenraadt et al., 2014). This study found increased prefrontal fNIRS signal during precision stepping with no change in the sensorimotor cortex.

Two studies implemented verbal tasks of various difficulties (Hill et al., 2013; Mirelman et al., 2014) and observed increased prefrontal fNIRS activity with increased task difficulty. Hill et al. (2013) compared serially counting backward by 1 with serially counting backward by 7 whereas Mirelman et al. (2014) compared counting forward with serially counting backward by 7 . Introducing different levels of task difficulty for a similar task enables a more robust assessment of cortical activity as the verbal motion artefact will be common to all graded tasks. Any significant change in the fNIRS signal observed between graded tasks is therefore more likely to be due to changes in cortical activity lending greater confidence in findings.

\subsubsection{Influence of ageing and $P D$}

Discrepancies were found between studies investigating the influence of age. Beurskens et al. (2014) recorded decreased prefrontal fNIRS signals in older adults during dual task walking. By contrast, Holtzer et al. (2011) and Fraser et al. (2016) found a comparable increase in prefrontal fNIRS signal from usual to dual task walking in young and older adults. Differences may firstly be accounted for by the different tasks performed. Beurskens et al. (2014) task was non-verbal which introduces less artefact than a verbal task. Different processing methods were involved with Beurskens et al. (2014) applying moving standard deviation and a precolouring filter whereas Holtzer et al. (2011) used a low pass filter and ICA/PCA. No processing method was reported by Fraser et al. (2016). Additionally, Beurskens et al. (2014) used small interoptode distances of $22 \mathrm{~mm}$ and $25 \mathrm{~mm}$, resulting in cortical tissue at an approximate depth of $11 \mathrm{~mm}-12.5 \mathrm{~mm}$ being probed (Scholkmann and Wolf, 2012). There may have been insufficient penetration of infra-red to sufficiently assess haemoglobin changes as the depth of the frontal cortex has been reported as $9.7 \mathrm{~mm}-17.1 \mathrm{~mm}$ with the superficial $10 \%$ of cortex having low vasculature density (Lauwers et al., 2008; Stokes et al., 2005). Brain morphology and cortical thickness change with age, which may further confound fNIRS findings between age groups (Provencher et al., 2016). Another consideration is the Beurskens et al. (2014) and Fraser et al. (2016) studies involved treadmill walking whereas Holtzer et al. (2016) examined overground walking. Finally, the number of older adults included in the three studies were small, numbering 10 (Beurskens et al., 2014), 14 (Fraser et al., 2016) and 11 (Holtzer et al., 2011), which may explain the diverse findings given the heterogeneity of older adults. No firm conclusion can be drawn regarding age-related changes in prefrontal cortical activity as too few studies have been undertaken with differing protocols and processing methods.

Three reviewed studies investigated fNIRS during walking in people with PD. One study reported higher prefrontal fNIRS signals when compared with older adults during usual walking but no change during cognitive dual task walking (Maidan et al., 2016). However, a greater prefrontal fNIRS signal was measured during obstacle negotiation 
(Maidan et al., 2016). Nieuwhof et al. (2016) reported no difference between three different tasks which may be the result of investigating a small sample of people with PD $(n=12)$ who exhibited very variable prefrontal activity.

One consideration when comparing different groups is how changes in cerebral properties will affect light propagation by altering absorption coefficients and DPF. Age-related cerebral changes including brain atrophy, small vessel disease, white matter hyperintensities, cerebral infarcts, Lewy bodies, neuritic plaques and neurofibrillary tangles (Rosso et al., 2013) will modify the DPF (Scholkmann and Wolf, 2013). Studies report an increase in DPF with age, although there are large individual differences (Duncan et al., 1996); (Scholkmann and Wolf, 2013). DPF values have however only been determined up to the age of 50 , when only small changes in cerebral structure have developed (Duncan et al., 1996). None of the sixteen reviewed studies investigating the effect of ageing on fNIRS activity reported the DPF values used. The depth of the cortex will also affect the DPF, with considerable intersubject variability and differences between scalp locations having been reported (Nakamura et al., 2016). The effect of age related cortical thinning and grey matter atrophy may increase the cortical depth in addition to increasing the amount of cerebrospinal fluid in the subarachnoid space which would affect light attenuation (Purdon et al., 2015). Changes in cortical microstructure in individuals with PD have been recorded which may modify light propagation (Nürnberger et al., 2017). Currently, no adjustments are made for pathological changes when determining light intensity therefore caution must be applied when comparing results between and within groups.

\subsection{Additional considerations}

The present review is limited to the information provided within the reviewed studies. However, additional factors that are pertinent include duration of the experiment, perspiration, pain from donning the fNIRS band or cap and hair (Kassab et al., 2015). Long experiments will increase both perspiration and pain. Perspiration may affect optode stability causing increased optode displacement and changes in orientation, adversely affecting the signal. Long periods of wearing the headband or cap may also result in external compression headache (Krymchantowski, 2010) and associated vasodilation, increased perspiration and possible oedema thereby further contaminating the signal (Schlereth and Birklein, 2008). The presence and type of hair (colour, thickness, density) underlying optodes can generate large motion artefacts through reflection of light and increased decoupling of the optodes from the scalp (Pringle et al., 1999; Yamada et al., 2015).

\subsection{Recommendations}

To date, studies using fNIRS have started to add interesting insights into the cortical control of walking, revealing important age and pathological changes that could inform future interventions. These studies also however, highlight the considerable technical and methodological challenges facing researchers in this field. Therefore, considering the evidence gathered from the reviewed studies, we make the following recommendations for future studies of cortical activity during gait:

Study design and operationalisation

- Sample sizes should be sufficiently large and justified by power analyses based on outcome measures recorded in pilot studies for a specific study protocol.

- Employ standardised block design protocols to control for time dependent effects relating to stimulus/experimental manipulation (for example asking a participant to walk for a set period rather than a given distance). This reduces need for retrospective signal processing (e.g. to normalise the signal length) avoiding signal distortion.

- Allow for the 4-7 s delay in haemodynamic peak response by including sufficiently long walks (/periods of exposure).
- Develop cognitive tasks with levels of difficulty that introduce minimal artefact (constant across levels of difficulty).

- Monitor systemic haemodynamics and incorporate short separation channels in optode arrangements to measure changes in scalp vasculature.

- Interoptode separation should be sufficiently large to adequately assess cortical bloodflow, with a minimum distance of $30 \mathrm{~mm}$ (Herold et al., 2017).

- Duration of the experiment and discomfort reported by the patient should be recorded.

\section{Signal processing analysis}

- Perform baseline correction trials before every trial to account for time-dependent changes in cerebral oxygenation.

- Report all steps of signal processing, including filtering and noise removal, and study specific processing or analysis.

- Signal processing techniques should be tailored according to the design protocol focussing on the nature of motion artefact present with regard to verbal and/or walking components with wavelet filtering applied to verbal task fNIRS signals (Brigadoi et al., 2014; Cooper et al., 2012).

- Adjust for baseline walking velocity in the analysis as a confounding factor.

- Algorithmsto determine haemoglobin concentration levels should be described and the DPF values provided.

- Future studies should aim to determine the effect of age related cerebral changes on DPF factor.

- Further work is required to fully appreciate motion artefact. Suggested studies include: using a phantom head to model the motion artefact in fNIRS; understanding he interaction between walking speed and motion artefact and how artefact is affected by wireless $v s$. tethered systems.

\section{Conclusions}

The 31 reviewed studies used a wide range of protocols and signal processing techniques which makes comparisons between studies difficult. Many studies involved small sample sizes which were not justified by power analysis. Length of data was often short and studies generally did not account for the haemodynamic response time-lag. Although most investigations concluded that there was greater cortical activity during usual walking, it is difficult to exclude confounding due to motion artefacts. Similarly, cognitive tasks which showed increased activity, mostly involved the subject vocalising during the task which introduces further artefact. Few studies applied wavelet filtering or used different levels of the cognitive task, which would add weight to the argument that increase in fNIRS activity is due to increased cortical activity rather than increased artefact. In summary, careful reporting of study methodologies will enhance reliability and confidence in the use of fNIRS to study cortical activity during walking.

\section{Conflicts of interest}

None.

\section{Acknowledgements}

This research is supported by the National Institute for Health Research (NIHR) Newcastle Biomedical Research Unit (BRU) and Centre (BRC) based at Newcastle upon Tyne Hospitals NHS Foundation Trust and Newcastle University. The research was also supported by NIHR Newcastle CRF Infrastructure funding and Sao Paulo Research Foundation (FAPESP, 2016/00518-8 as a postdoctoral fellowship award for Rodrigo Vitorio). The views expressed are those of the authors and not necessarily those of the NHS, the NIHR or the Department of Health. 


\section{References}

Atsumori, H., Kiguchi, M., Katura, T., Funane, T., Obata, A., Sato, H., Manaka, T., Iwamoto, M., Maki, A., Koizumi, H., Kubota, K., 2010. Noninvasive imaging of prefrontal activation during attention-demanding tasks performed while walking using a wearable optical topography system. J. Biomed. Opt. 15, 046002.

Bakker, M., De Lange, F.P., Helmich, R.C., Scheeringa, R., Bloem, B.R., Toni, I., 2008. Cerebral correlates of motor imagery of normal and precision gait. Neuroimage 41, 998-1010.

Beurskens, R., Helmich, I., Rein, R., Bock, O., 2014. Age-related changes in prefrontal activity during walking in dual-task situations: a fNIRS study. Int. J. Psychophysiol. 92, 122-128.

Boas, D.A., Dale, A.M., Franceschini, M.A., 2004. Diffuse optical imaging of brain activation: approaches to optimizing image sensitivity, resolution, and accuracy Neuroimage 23 (Suppl. 1), S275-288.

Brigadoi, S., Ceccherini, L., Cutini, S., Scarpa, F., Scatturin, P., Selb, J., Gagnon, L., Boas, D.A., Cooper, R.J., 2014. Motion artifacts in functional near-infrared spectroscopy: a comparison of motion correction techniques applied to real cognitive data. Neuroimage 85 (Pt 1), 181-191.

Chiarelli, A.M., Maclin, E.L., Fabiani, M., Gratton, G., 2015. A kurtosis-based wavelet algorithm for motion artifact correction of fNIRS data. Neuroimage 112 128-137.

Clark, D.J., Christou, E.A., Ring, S.A., Williamson, J.B., Doty, L., 2014a. Enhanced somatosensory feedback reduces prefrontal cortical activity during walking in older adults. J. Gerontol. A Biol. Sci. Med. Sci. 69, 1422-1428.

Clark, D.J., Rose, D.K., Ring, S.A., Porges, E.C., 2014b. Utilization of central nervous system resources for preparation and performance of complex walking tasks in older adults. Front. Aging Neurosci. 6, 217.

Cooper, R.J., Selb, J., Gagnon, L., Phillip, D., Schytz, H.W., Iversen, H.K., Ashina, M., Boas, D.A., 2012. A systematic comparison of motion artifact correction techniques for functional near-infrared spectroscopy. Front. Neurosci. 6, 147.

Cui, X., Bray, S., Reiss, A.L., 2010. Speeded near infrared spectroscopy (NIRS) response detection. PLoS One 5, e15474.

Cui, X., Bray, S., Bryant, D.M., Glover, G.H., Reiss, A.L., 2011. A quantitative comparison of NIRS and fMRI across multiple cognitive tasks. Neuroimage 54, 2808-2821.

Cutini, S., Scarpa, F., Scatturin, P., Jolicœur, P., Pluchino, P., Zorzi, M., Dell'Acqua, R., 2011. A hemodynamic correlate of lateralized visual short-term memories. Neuropsychologia 49, 1611-1621.

Deppermann, S., Vennewald, N., 2014. Does rTMS alter neurocognitive functioning in patients with panic disorder/agoraphobia? An fNIRS-based Investigation of Prefrontal Activation During a Cognitive Task and Its Modulation via Sham-controlled rTMS. pp. 542526.

Doi, T., Makizako, H., Shimada, H., Park, H., Tsutsumimoto, K., Uemura, K., Suzuki, T., 2013. Brain activation during dual-task walking and executive function among older adults with mild cognitive impairment: a fNIRS study. Aging Clin. Exp. Res. 25, 539-544.

Duncan, A., Meek, J.H., Clemence, M., Elwell, C.E., Tyszczuk, L., Cope, M., Delpy, D., 1995. Optical pathlength measurements on adult head, calf and forearm and the head of the newborn infant using phase resolved optical spectroscopy. Phys. Med. Biol. 40, 295.

Duncan, A., Meek, J.H., Clemence, M., Elwell, C.E., Fallon, P., Tyszczuk, L., Cope, M., Delpy, D.T., 1996. Measurement of cranial optical path length as a function of age using phase resolved near infrared spectroscopy. Pediatr. Res. 39, 889-894.

Eggenberger, P., Wolf, M., Schumann, M., de Bruin, E.D., 2016. Exergame and balance training modulate prefrontal brain activity during walking and enhance executive function in older adults. Front. Aging Neurosci. 8.

Ferrari, M., Quaresima, V., 2012. A brief review on the history of human functional nearinfrared spectroscopy (fNIRS) development and fields of application. Neuroimage 63, 921-935.

Fraser, S.A., Dupuy, O., Pouliot, P., Lesage, F., Bherer, L., 2016. Comparable cerebral oxygenation patterns in younger and older adults during dual-Task walking with increasing load. Front. Aging Neurosci. 8, 240.

Fujimoto, H., Mihara, M., Hattori, N., Hatakenaka, M., Kawano, T., Yagura, H., Miyai, I., Mochizuki, H., 2014. Cortical changes underlying balance recovery in patients with hemiplegic stroke. Neuroimage 85, 547-554.

Galna, B., Lord, S., Burn, D.J., Rochester, L., 2015. Progression of gait dysfunction in incident Parkinson's disease: impact of medication and phenotype. Mov. Disord. 30, 359-367.

Hamacher, D., Herold, F., Wiegel, P., Hamacher, D., Schega, L., 2015. Brain activity during walking: a systematic review. Neurosci. Biobehav. Rev. 57, 310-327.

Harada, T., Miyai, I., Suzuki, M., Kubota, K., 2009. Gait capacity affects cortical activation patterns related to speed control in the elderly. Exp. Brain Res. 193, 445-454.

Hernandez, M.E., Holtzer, R., Chaparro, G., Jean, K., Balto, J.M., Sandroff, B.M., Izzetoglu, M., Motl, R.W., 2016. Brain activation changes during locomotion in middle-aged to older adults with multiple sclerosis. J. Neurol. Sci. 370, 277-283.

Herold, F., Wiegel, P., Scholkmann, F., Thiers, A., Hamacher, D., Schega, L., 2017. Functional near-infrared spectroscopy in movement science: a systematic review on cortical activity in postural and walking tasks. Neurophotonics 4 lt;xocs:firstpage xmlns:xocs = "'"/ \& gt.

Hill, A., Bohil, C., Lewis, J., Neider, M.a., 2013. Prefrontal cortex activity during walking while multitasking: an fNIR study. HumanFactors and Ergonomic Society 57th Annual Meeting.

Holper, L., Scholkmann, F., Shalóm, D.E., Wolf, M., 2012. Extension of mental preparation positively affects motor imagery as compared to motor execution: a functional near-infrared spectroscopy study. Cortex 48, 593-603.
Holtzer, R., Mahoney, J.R., Izzetoglu, M., Izzetoglu, K., Onaral, B., Verghese, J., 2011. fNIRS study of walking and walking while talking in young and old individuals. J. Gerontol. A Biol. Sci. Med. Sci. 66, 879-887.

Holtzer, R., Epstein, N., Mahoney, J.R., Izzetoglu, M., Blumen, H.M., 2014. Neuroimaging of mobility in aging: a targeted review. J. Gerontol. A Biol. Sci. Med. Sci. 69, 1375-1388.

Holtzer, R., Mahoney, J.R., Izzetoglu, M., Wang, C., England, S., Verghese, J., 2015. Online fronto-cortical control of simple and attention-demanding locomotion in humans. Neuroimage 112, 152-159.

Holtzer, R., Verghese, J., Allali, G., Izzetoglu, M., Wang, C., Mahoney, J.R., 2016. Neurological gait abnormalities moderate the functional brain signature of the posture first hypothesis. Brain Topogr. 29, 334-343.

Holtzer, R., Schoen, C., Demetriou, E., Mahoney, J.R., Izzetoglu, M., Wang, C., Verghese, J., 2017a. Stress and gender effects on prefrontal cortex oxygenation levels assessed during single and dual-task walking conditions. Eur. J. Neurosci. 45, 660-670.

Holtzer, R., Yuan, J., Verghese, J., Mahoney, J.R., Izzetoglu, M., Wang, C., 2017b. Interactions of subjective and objective measures of fatigue defined in the context of brain control of locomotion. J. Gerontol. A Biol. Sci. Med. Sci. 72, 417-423.

Huppert, T., Schmidt, B., Beluk, N., Furman, J., Sparto, P., 2013. Measurement of brain activation during an upright stepping reaction task using functional near-infrared spectroscopy. Hum. Brain Mapp. 34, 2817-2828.

Izzetoglu, M., Bunce, S.C., Izzetoglu, K., Onaral, B., Pourrezaei, K., 2007. Functional brain imaging using near-infrared technology. IEEE Eng. Med. Biol. Mag. 26, 38-46.

Kamran, M.A., Mannan, M.M., Jeong, M.Y., 2016. Cortical signal analysis and advances in functional near-Infrared spectroscopy signal: a review. Front. Hum. Neurosci. 10, 261.

Karim, H., Schmidt, B., Dart, D., Beluk, N., Huppert, T., 2012. Functional near-infrared spectroscopy (fNIRS) of brain function during active balancing using a video game system. Gait Posture 35, 367-372.

Kassab, A., Le Lan, J., Vannasing, P., Sawan, M., 2015. Functional near-infrared spectroscopy caps for brain activity monitoring: a review. Appl. Opt. 54, 576-586.

Kawai, N., Kubo-Kawai, N., Kubo, K., Terazawa, T., Masataka, N., 2012. Distinct Aging Effects for Two Types of Inhibition in Older Adults: A Near-infrared Spectroscopy Study on the Simon Task and the Flanker Task. Lippincott Williams and Wilkins, United Kingdom, pp. 819-824 (250 Waterloo Road, London SE1 8RD, United Kingdom).

Khan, M.J., Hong, K.-S., 2015. Passive BCI based on drowsiness detection: an fNIRS study. Biomed. Opt. Express 6, 4063-4078.

Kim, H.Y., Yang, S.P., Park, G.L., Kim, E.J., You, J.S., 2016. Best facilitated cortical activation during different stepping, treadmill, and robot-assisted walking training paradigms and speeds: a functional near-infrared spectroscopy neuroimaging study. NeuroRehabilitation 38, 171-178.

Kirilina, E., Jelzow, A., Heine, A., Niessing, M., Wabnitz, H., Bruhl, R., Ittermann, B., Jacobs, A.M., Tachtsidis, I., 2012. The physiological origin of task-evoked systemic artefacts in functional near infrared spectroscopy. Neuroimage 61, 70-81.

Kline, J.E., Huang, H.J., Snyder, K.L., Ferris, D.P., 2015. Isolating gait-related movement artifacts in electroencephalography during human walking. J. Neural Eng. 12, 046022.

Koenraadt, K.L., Roelofsen, E.G., Duysens, J., Keijsers, N.L., 2014. Cortical control of normal gait and precision stepping: an fNIRS study. Neuroimage 85 (Pt 1), 415-422.

Krymchantowski, A.V., 2010. Headaches due to external compression. Curr. Pain Headache Rep. 14, 321-324.

Kurz, M.J., Wilson, T.W., Arpin, D.J., 2012. Stride-time variability and sensorimotor cortical activation during walking. Neuroimage 59, 1602-1607.

Lareau, E., Lesage, F., Pouliot, P., Nguyen, D., Le Lan, J., Sawan, M., 2011. Multichannel wearable system dedicated for simultaneous electroencephalographynear-infrared spectroscopy real-time data acquisitions. J. Biomed. Opt. 16.

Lauwers, F., Cassot, F., Lauwers-Cances, V., Puwanarajah, P., Duvernoy, H., 2008. Morphometry of the human cerebral cortex microcirculation: general characteristics and space-related profiles. Neuroimage 39, 936-948.

Lin, M.I.B., Lin, K.H., 2016. Walking while performing working memory tasks changes the prefrontal cortex hemodynamic activations and gait kinematics. Front. Behav. Neurosci. 10, 92. http://dx.doi.org/10.3389/fnbeh.2016.00092.

Lord, S., Galna, B., Coleman, S., Yarnall, A., Burn, D., Rochester, L., 2014. Cognition and gait show a selective pattern of association dominated by phenotype in incident Parkinson's disease. Front. Aging Neurosci. 6, 249.

Lord, S., Galna, B., Yarnall, A.J., Coleman, S., Burn, D., Rochester, L., 2016. Predicting first fall in newly diagnosed Parkinson's disease: insights from a fall-naive cohort. Mov. Disord. 31, 1829-1836.

Lu, C.F., Liu, Y.C., Yang, Y.R., Wu, Y.T., Wang, R.Y., 2015. Maintaining gait performance by cortical activation during dual-Task interference: a functional near-Infrared spectroscopy study. PLoS One 10, e0129390.

Mahoney, J.R., Holtzer, R., Izzetoglu, M., Zemon, V., Verghese, J., Allali, G., 2016. The role of prefrontal cortex during postural control in Parkinsonian syndromes a functional near-infrared spectroscopy study. Brain Res. 1633, 126-138.

Maidan, I., Bernad-Elazari, H., Gazit, E., Giladi, N., Hausdorff, J.M., Mirelman, A., 2015 Changes in oxygenated hemoglobin link freezing of gait to frontal activation in patients with Parkinson disease: an fNIRS study of transient motor-cognitive failures. J. Neurol. 262, 899-908.

Maidan, I., Nieuwhof, F., Bernad-Elazari, H., Reelick, M., Bloem, B., Giladi, N., Deutsch, J., Hausdorff, J., Claassen, J., Mirelman, A., 2016. The role of the frontal lobe in complex walking among healthy older adults and patients with Parkinson's disease: an fNIRS study. In: 20th International Congress of Parkinson's Disease and Movement Disorders. Berlin.

Maki, A., Yamashita, Y., Ito, Y., Watanabe, E., Mayanagi, Y., Koizumi, H., 1995. Spatial and temporal analysis of human motor activity using noninvasive NIR topography. 
Med. Phys. 22, 1997-2005.

Meester, D., Al-Yahya, E., Dawes, H., Martin-Fagg, P., Pinon, C., 2014. Associations between prefrontal cortex activation and H-reflex modulation during dual task gait. Front. Hum. Neurosci. 8, 78 .

Metzger, F.G., Ehlis, A.C., Haeussinger, F.B., Schneeweiss, P., Hudak, J., Fallgatter, A.J., Schneider, S., 2017. Functional brain imaging of walking while talking - An fNIRS study. Neuroscience 343, 85-93.

Mihara, M., Miyai, I., Hatakenaka, M., Kubota, K., Sakoda, S., 2007. Sustained prefrontal activation during ataxic gait: a compensatory mechanism for ataxic stroke? Neuroimage 37, 1338-1345.

Mihara, M., Miyai, I., Hatakenaka, M., Kubota, K., Sakoda, S., 2008. Role of the prefrontal cortex in human balance control. Neuroimage 43, 329-336.

Mirelman, A., Maidan, I., Bernad-Elazari, H., Nieuwhof, F., Reelick, M., Giladi, N., Hausdorff, J.M., 2014. Increased frontal brain activation during walking while dual tasking: an fNIRS study in healthy young adults. J. Neuroeng. Rehabil. 11, 85.

Miyai, I., Tanabe, H.C., Sase, I., Eda, H., Oda, I., Konishi, I., Tsunazawa, Y., Suzuki, T., Yanagida, T., Kubota, K., 2001. Cortical mapping of gait in humans: a near-infrared spectroscopic topography study. Neuroimage 14, 1186-1192.

Montero-Odasso, M., Verghese, J., Beauchet, O., Hausdorff, J.M., 2012. Gait and cognition: a complementary approach to understanding brain function and the risk of falling. J. Am. Geriatr. Soc. 60, 2127-2136.

Nürnberger, L., Gracien, R.-M., Hok, P., Hof, S.-M., Rüb, U., Steinmetz, H., Hilker, R., Klein, J.C., Deichmann, R., Baudrexel, S., 2017. Longitudinal changes of cortical microstructure in Parkinson's disease assessed with T1 relaxometry. NeuroImage.Clin. 13, 405-414.

Nakamura, K., Kurihara, K., Kawaguchi, H., Obata, T., Ito, H., Okada, E., 2016. Estimation of partial optical path length in the brain in subject-specific head models for nearinfrared spectroscopy. Opt. Rev. 23, 316-322.

Nieuwhof, F., Reelick, M.F., Maidan, I., Mirelman, A., Hausdorff, J.M., Olde Rikkert, M.G., Bloem, B.R., Muthalib, M., Claassen, J.A., 2016. Measuring Prefrontal Cortical Activity During Dual Task Walking in Patients with Parkinson's Disease: Feasibility of Using a New Portable fNIRS Device. Pilot Feasibility Stud 259.

Niu, H.J., Li, X., Chen, Y.J., Ma, C., Zhang, J.Y., Zhang, Z.J., 2013. Reduced frontal activation during a working memory task in mild cognitive impairment: a non-invasive near-infrared spectroscopy study. CNS Neurosci. Ther. 19, 125-131.

Ono, Y., Nomoto, Y., Tanaka, S., Sato, K., Shimada, S., Tachibana, A., Bronner, S., Noah, J.A., 2014. Frontotemporal oxyhemoglobin dynamics predict performance accuracy of dance simulation gameplay: temporal characteristics of top-down and bottom-up cortical activities. Neuroimage 85, 461-470.

Orihuela-Espina, F., Leff, D.R., James, D.R., Darzi, A.W., Yang, G.Z., 2010. Quality control and assurance in functional near infrared spectroscopy (fNIRS) experimentation. Phys. Med. Biol. 55, 3701-3724.

Osofundiya, O., Benden, M.E., Dowdy, D., Mehta, R.K., 2016. Obesity-specific neural cost of maintaining gait performance under complex conditions in community-dwelling older adults. Clin. Biomech. (Bristol, Avon) 35, 42-48.

Parvataneni, K., Ploeg, L., Olney, S.J., Brouwer, B., 2009. Kinematic, kinetic and metabolic parameters of treadmill versus overground walking in healthy older adults. Clin. Biomech. (Bristol, Avon) 24, 95-100.

Pinti, P., Aichelburg, C., Lind, F., Power, S., Swingler, E., Merla, A., Hamilton, A., Gilber, S., Burgess, P., Tachtsidis, I., 2015. Using fiberless, wearable fnirs to monitor brain activity in real-world cognitive tasks. J. Visualized Exp. 2015.

Piper, S.K., Krueger, A., Koch, S.P., Mehnert, J., Habermehl, C., Steinbrink, J., Obrig, H., Schmitz, C.H., 2014. A wearable multi-channel fNIRS system for brain imaging in freely moving subjects. Neuroimage 85, 64-71.

Pringle, J., Roberts, C., Kohl, M., Lekeux, P., 1999. Near infrared spectroscopy in large animals: optical pathlength and influence of hair covering and epidermal pigmentation. Vet. J. 158, 48-52.

Provencher, D., Hennebelle, M., Cunnane, S.C., Bérubé-Lauzière, Y., Whittingstall, K., 2016. Cortical thinning in healthy aging correlates with larger motor-Evoked EEG desynchronization. Front. Aging Neurosci. 8, 63

Purdon, P.L., Pavone, K.J., Akeju, O., Smith, A.C., Sampson, A.L., Lee, J., Zhou, D.W., Solt, K., Brown, E.N., 2015. The Ageing Brain: age-dependent changes in the electroencephalogram during propofol and sevoflurane general anaesthesia. BJA Br. J. Anaesthesia 115, i46-i57.

Riley, P.O., Paolini, G., Della Croce, U., Paylo, K.W., Kerrigan, D.C., 2007. A kinematic and kinetic comparison of overground and treadmill walking in healthy subjects. Gait Posture 26, 17-24.

Rosso, A.L., Studenski, S.A., Chen, W.G., Aizenstein, H.J., Alexander, N.B., Bennett, D.A., Black, S.E., Camicioli, R., Carlson, M.C., Ferrucci, L., Guralnik, J.M., Hausdorff, J.M.,
Kaye, J., Launer, L.J., Lipsitz, L.A., Verghese, J., Rosano, C., 2013. Aging, the central nervous system, and mobility. J. Gerontol. A. Biol. Sci. Med. Sci. 68, 1379-1386.

Ruocco, A.C., Rodrigo, A.H., Carcone, D., McMain, S., Jacobs, G., Kennedy, J.L., 2016. Tryptophan hydroxylase 1 gene polymorphisms alter prefrontal cortex activation during response inhibition. Am. Psychol. Asso. Inc. U. S. 18-27.

Schlereth, T., Birklein, F., 2008. The sympathetic nervous system and pain. Neuromol. Med. 10, 141-147.

Scholkmann, F., Wolf, M.H., 2012. Measuring brain activity using functional ear infrared spectroscopy:A short review. Spectrosc. Eur. 24, 6-10.

Scholkmann, F., Wolf, M., 2013. General equation for the differential pathlength factor of the frontal human head depending on wavelength and age. J. Biomed. Opt. 18, 105004.

Scholkmann, F., Spichtig, S., Muehlemann, T., Wolf, M., 2010. How to detect and reduce movement artifacts in near-infrared imaging using moving standard deviation and spline interpolation. Physiol. Meas. 31, 649-662.

Scholkmann, F., Gerber, U., Wolf, M., Wolf, U., 2013. End-tidal CO2: an important parameter for a correct interpretation in functional brain studies using speech tasks. Neuroimage 66, 71-79.

Scholkmann, F., Kleiser, S., Metz, A.J., Zimmermann, R., Mata Pavia, J., Wolf, U., Wolf, M., 2014. A review on continuous wave functional near-infrared spectroscopy and imaging instrumentation and methodology. Neuroimage 85 (Pt 1), 6-27.

Scholkmann, F., Klein, S.D., Gerber, U., Wolf, M., Wolf, U., 2014a. Cerebral hemodynamic and oxygenation changes induced by inner and heard speech: a study combining functional near-infrared spectroscopy and capnography. J. Biomed. Opt. 19, 17002

Shumway-Cook, A., Woollacott, M.H., 2007. Motor Control: Translating Research into Clinical Practice, 3rd ed. Lippincott Williams \& Wilkins.

Stevens, J.A., Corso, P.S., Finkelstein, E.A., Miller, T.R., 2006. The costs of fatal and nonfatal falls among older adults. Inj. Prev. 12, 290-295.

Stokes, M.G., Chambers, C.D., Gould, I.C., Henderson, T.R., Janko, N.E., Allen, N.B., Mattingley, J.B., 2005. Simple metric for scaling motor threshold based on scalpCortex distance: application to studies using transcranial magnetic stimulation. J. Neurophysiol. 94, 4520-4527.

Strangman, G., Culver, J.P., Thompson, J.H., Boas, D.A., 2002. A quantitative comparison of simultaneous BOLD fMRI and NIRS recordings during functional brain activation. Neuroimage 17, 719-731.

Suzuki, M., Miyai, I., Ono, T., Oda, I., Konishi, I., Kochiyama, T., Kubota, K., 2004. Prefrontal and premotor cortices are involved in adapting walking and running speed on the treadmill: an optical imaging study. Neuroimage 23, 1020-1026.

Suzuki, M., Miyai, I., Ono, T., Kubota, K., 2008. Activities in the frontal cortex and gait performance are modulated by preparation. fNIRS Study NeuroImage 39, 600-607.

Takahashi, T., Takikawa, Y., Kawagoe, R., Shibuya, S., Iwano, T., Kitazawa, S., 2011. Influence of skin blood flow on near-infrared spectroscopy signals measured on the forehead during a verbal fluency task. Neuroimage 57, 991-1002.

Tong, Y., Frederick, B.D., 2010. Time lag dependent multimodal processing of concurrent fMRI and near-infrared spectroscopy (NIRS) data suggests a global circulatory origin for low-frequency oscillation signals in human brain. Neuroimage 53, 553-564.

Verghese, J., Wang, C., Ayers, E., Izzetoglu, M., Holtzer, R., 2017. Brain activation in high-functioning older adults and falls: prospective cohort study. Neurology 88, 191-197.

Vitorio, R., Lirani-Silva, E., Baptista, A.M., Barbieri, F.A., dos Santos, P.C., TeixeiraArroyo, C., Gobbi, L.T., 2014. Disease severity affects obstacle crossing in people with Parkinson's disease. Gait Posture 40, 266-269.

Wriessnegger, S.C., Bauernfeind, G., Schweitzer, K., Kober, S., Neuper, C., Müller-Putz, G.R., 2012. The interplay of prefrontal and sensorimotor cortices during inhibitory control of learned motor behaviour. Front. Neuroeng. 5http://dx.doi.org/10.3389/ fneng.2012.00017. Article 17.

Yamada, T., Umeyama, S., Ohashi, M., 2015. Removal of motion artifacts originating from optode fluctuations during functional near-infrared spectroscopy measurements. Biomed. Opt. Express 6, 4632-4649.

Yuan, P., Raz, N., 2014. Prefrontal cortex and executive functions in healthy adults: a meta-analysis of structural neuroimaging studies. Neurosci. Biobehav. Rev. 42, 180-192.

la Fougere, C., Zwergal, A., Rominger, A., Forster, S., Fesl, G., Dieterich, M., Brandt, T. Strupp, M., Bartenstein, P., Jahn, K., 2010. Real versus imagined locomotion: a [18F] FDG PET-fMRI comparison. Neuroimage 50, 1589-1598.

von Luhmann, A., 2014. Design and evaluation of a system for mobile brain activity measurements using functional near-Infrared spectroscopy. Inst. Biomed. Eng. Karlsruhe Inst. Technol. 